\title{
Multislice Spiral Computed Tomography Coronary Angiography in Patients With Stable Angina Pectoris
}

\author{
Nico R. Mollet, MD, ${ }^{*}+$ Filippo Cademartiri, MD, $\dagger$ Koen Nieman, MD, ${ }^{*}$ Francesco Saia, MD,*
} Pedro A. Lemos, MD, ${ }^{*}$ Eugene P. McFadden, MB, ${ }^{*}$ Peter M. T. Pattynama, MD, $\dagger$ Patrick W. Serruys, MD, FACC,* Gabriel P. Krestin, MD, $†$ Pim J. de Feyter, MD, FACC*† Rotterdam, Netherlands

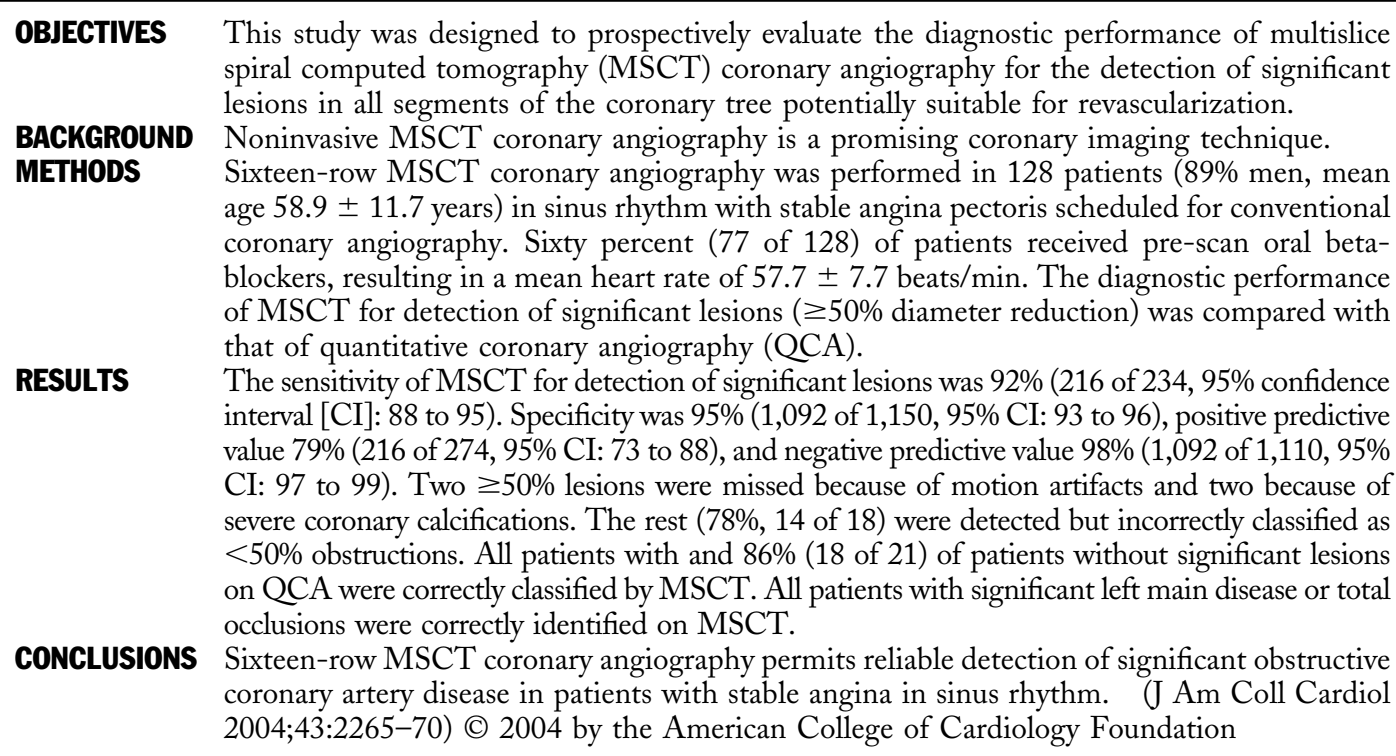

Multislice spiral computed tomography (MSCT) coronary angiography is a promising noninvasive technique for the detection of obstructive epicardial coronary artery disease (CAD), and 16-row MSCT scanners have the potential to further improve its diagnostic performance (1). Complete visualization of all clinically important coronary segments is a prerequisite for MSCT coronary angiography to become an accepted clinical tool for the assessment of patients with suspected CAD. Previous studies performed in relatively small numbers of patients showed high sensitivity and specificity for the detection of significant obstructive lesions $(2,3)$. However, in one study, a significant number of coronary segments were excluded because of poor image quality, and neither report presented a comprehensive lesion-by-lesion comparative analysis.

We prospectively evaluated the diagnostic accuracy of MSCT coronary angiography for the detection of significant lesions in coronary segments potentially amenable to revascularization ( $\geq 2 \mathrm{~mm}$ in diameter).

\section{METHODS}

Study population. During a period of six months, we studied 128 patients (113 men, 15 women, mean age $58.9 \pm$

From the *Department of Cardiology, Thoraxcenter; and the $\dagger$ Department of Radiology, Erasmus Medical Center, Rotterdam, Netherlands.

Manuscript received December 18, 2003; revised manuscript received January 22, 2004, accepted March 2, 2004
11.7 years) with stable angina pectoris scheduled for conventional coronary angiography. Only patients in sinus rhythm able to perform a 20 -s breath-hold were included. Exclusion criteria were contraindications to iodinated contrast (e.g., known allergy, serum creatinine $>120 \mathrm{mmol} / \mathrm{l}$, and thyroid disorders), patients with previous bypass surgery, and patients presenting with an acute coronary syndrome. No patients of the previously reported studies were included in the present study.

The institutional review board of our institution approved the study and all patients gave written, informed consent.

Patient preparation. A single oral dose of $100 \mathrm{mg}$ metoprolol (Selokeen, AstraZeneca Pharmaceutics, London, United Kingdom) was administered $1 \mathrm{~h}$ before the scan if the heart rate was $\geq 65$ beats $/ \mathrm{min}$.

Scan protocol and image reconstruction. All scans were performed using a 16-row MSCT scanner (Sensation 16, Siemens, Germany). Scan parameters were: detector collimation $16 \times 0.75 \mathrm{~mm}$, tube rotation time $420 \mathrm{~ms}$, table feed $3 \mathrm{~mm} /$ rotation, tube voltage $120 \mathrm{kV}$, and tube current 400 to $450 \mathrm{mAs}$. Prospectively triggered X-ray tube current modulation was applied in patients with a heart rate below 60 beats/min and in the absence of any rhythm disturbances during a monitoring period of approximately $1 \mathrm{~min}$. This feature reduces the radiation output of the $\mathrm{X}$-tube during the less important systolic phase, thereby decreasing the total radiation dose by $40 \%$ to $50 \%$ in patients with low heart rates $(4,5)$. A bolus of $100 \mathrm{ml}$ of contrast (Visipaque 
Abbreviations and Acronyms

$$
\begin{array}{ll}
\mathrm{CAD} & =\text { coronary artery disease } \\
\mathrm{CI} & =\text { confidence interval } \\
\mathrm{CT} & =\text { computed tomography } \\
\mathrm{MSCT} & =\text { multislice spiral computed tomography } \\
\text { QCA } & =\text { quantitative coronary angiography }
\end{array}
$$

320, Amersham Health, Forchheim, United Kingdom) was injected intravenously at a flow rate of $4 \mathrm{ml} / \mathrm{s}$. An automated bolus tracking system was used to synchronize the arrival of the contrast material with initiation of the scan. All data were acquired during a single breath-hold of approximately $20 \mathrm{~s}$, and images were reconstructed using retrospective ECG gating. To obtain motion-free images, standard reconstruction windows were selected during the mid-toend diastolic phase (350, 400, and $450 \mathrm{~ms}$ before the next $\mathrm{R}$-wave). Additional image reconstruction windows were explored when deemed necessary. The reconstruction algorithm uses data obtained in half gantry rotation time, resulting in a temporal resolution of up to $210 \mathrm{~ms}$. In case of a heart rate of $>70$ beats $/ \mathrm{min}$, a bi-segmental reconstruction algorithm is applied that uses data obtained from two consecutive heartbeats, reducing the effective reconstruction interval per heart cycle down to $105 \mathrm{~ms}$, depending on the heart rate (1).

Quantitative coronary angiography (QCA). The mean $( \pm \mathrm{SD})$ interval between the MSCT scan and conventional coronary angiography was $19.1 \pm 10.4$ days. The coronary arteries were divided into segments according to the American Heart Association classification (6). A single observer, unaware of the MSCT results, classified all coronary segments as $<2$ and $\geq 2 \mathrm{~mm}$ in diameter using a QCA algorithm (CAAS, Pie Medical, Maastricht, Netherlands). Only segments classified as $\geq 2 \mathrm{~mm}$ were considered for comparison with MSCT. The severity of coronary stenoses was quantified in two orthogonal views, and a stenosis was classified as significant if the mean lumen diameter reduction was $\geq 50 \%$.

MSCT image evaluation. Two observers blind to the results of conventional coronary angiography independently evaluated all of the MSCT scans. Thin-slab maximum intensity projections with a slice thickness of 2 to $6 \mathrm{~mm}$, depending on the presence of adjacent structures or coronary calcifications, were used to screen for coronary stenoses. Multiplanar reconstructions were used to obtain more detailed information.

All main branches as well as large ( $\geq 2 \mathrm{~mm}$ in lumen diameter) side branches of the coronary tree were evaluated for the presence of significant ( $\geq 50 \%$ diameter reduction) obstructive stenoses. Segments with stents were excluded from analysis because beam-hardening artifacts and partial volume effects hamper reliable visualization of the coronary lumen. Image quality of all segments was classified as good, adequate, or poor. Good image quality was classified as the absence of any image-degrading artifacts related to motion, noise, or calcification. In the presence of image-degrading artifacts, image quality was classified as adequate or poor. Adequate image quality allowed the assessment of significant lesions with moderate confidence, whereas poor image quality allowed the assessment of significant lesions with only a low confidence.

The presence of calcium in the coronary wall was systematically assessed. Each segment was classified as noncalcified, moderately calcified (small isolated eccentric highdensity lesions in the coronary wall), or heavily calcified (high-density lesions extending longitudinally along the coronary wall, causing beam hardening and partial volume artifacts). Disagreements were resolved by consensus.

Statistical analysis. The diagnostic performance of MSCT coronary angiography for the detection of significant obstructive lesions, with QCA as the standard of reference, is presented as sensitivity, specificity, and negative and positive predictive value. These diagnostic parameters are expressed with a $95 \%$ confidence interval calculated with binomial expansion. On a lesion-by-lesion analysis, inter- and intraobserver variability for the detection of significant lesions was calculated and expressed as kappa values. The most proximal significant stenosis was considered as the predominant lesion in the vessel-based analysis.

\section{RESULTS}

Sixty percent (77 of 128) of the patients received a betablocker before the MSCT scan and 42 of these patients were already receiving beta-blockers. The mean $( \pm \mathrm{SD})$ heart rate was $57.7 \pm 7.7$ during the scan procedure. The total scan time was $18.2 \pm 1.4 \mathrm{~s}$. One scan could not be evaluated because of technical failure. Prospectively triggered X-tube modulation was applied in 64\% (81/127) of the remaining patients.

Conventional coronary angiography revealed no significant stenoses in $17 \%$ (21 of 127), one-vessel disease in 35\% (44 of 127), two-vessel disease in 35\% (44 of 127), and three-vessel disease in 14\% (18 of 127) of patients. Six patients had significant left main stenosis.

Lesion-by-lesion analysis: overall performance of MSCT. A total of 1,384 non-stented segments with a diameter $\geq 2 \mathrm{~mm}$ were analyzed for the detection of significant obstructive coronary stenoses (37 segments with stents were excluded). Per patient, we included $10.9 \pm 1.9$ segments for analysis. Image quality was classified as good in $75 \%(1,049$ of 1,384$)$ of segments, adequate in $18 \%$ (243 of $1,384)$ of segments, and poor in 7\% (92 of 1,384) of segments. Causes of poorly assessable segments were motion artifacts $(63 \%, 58$ of 92), severe calcification $(30 \%, 28$ of 92), and low contrast-to-noise ratio (7\%, 6 of 92). Motion artifacts were most frequently located in the midright coronary artery. Inter- and intraobserver variability for the detection of significant lesions had kappa values of 0.71 and 0.79 , respectively.

The sensitivity was $92 \%$ (216 of $234,95 \%$ confidence 
Table 1. Results: Detection of Significant ( $\geq 50 \%)$ Stenoses With 16-Row Multislice Spiral Computed Tomography Coronary Angiography

\begin{tabular}{lrrrrccccc}
\hline Coronary Segment & N & TP & TN & FP & FN & Sensitivity & Specificity & Positive PV & Negative PV \\
\hline All segments & 1,384 & 216 & 1,092 & 58 & 18 & $92 \%(216 / 234)$ & $95 \%(1,092 / 1,150)$ & $79 \%(216 / 274)$ & $98 \%(1,092 / 1,110)$ \\
LM & 124 & 6 & 118 & 0 & 0 & $100 \%(6 / 6)$ & $100 \%(118 / 118)$ & $100 \%(6 / 6)$ & $100 \%(118 / 118)$ \\
LAD & 473 & 90 & 350 & 27 & 6 & $94 \%(90 / 96)$ & $93 \%(350 / 377)$ & $77 \%(90 / 117)$ & $98 \%(350 / 356)$ \\
$\quad$ Proximal & 124 & 37 & 74 & 10 & 3 & $93 \%(37 / 40)$ & $88 \%(74 / 84)$ & $79 \%(37 / 47)$ & $96 \%(350 / 353)$ \\
Middle & 111 & 41 & 65 & 5 & 0 & $100 \%(41 / 41)$ & $93 \%(65 / 70)$ & $89 \%(41 / 46)$ & $100 \%(65 / 65)$ \\
Distal & 102 & 5 & 94 & 3 & 0 & $100 \%(5 / 5)$ & $97 \%(94 / 97)$ & $63 \%(5 / 8)$ & $100 \%(94 / 94)$ \\
Side branches & 136 & 7 & 117 & 9 & 3 & $70 \%(7 / 10)$ & $93 \%(117 / 126)$ & $44 \%(7 / 16)$ & $98 \%(117 / 120)$ \\
CX & 395 & 49 & 325 & 12 & 9 & $84 \%(49 / 58)$ & $96 \%(325 / 337)$ & $80 \%(49 / 61)$ & $97 \%(325 / 334)$ \\
Proximal & 111 & 16 & 90 & 3 & 2 & $89 \%(16 / 18)$ & $97 \%(90 / 93)$ & $84 \%(16 / 19)$ & $98 \%(90 / 92)$ \\
Middle & 102 & 13 & 81 & 4 & 4 & $76 \%(13 / 17)$ & $95 \%(81 / 85)$ & $76 \%(13 / 17)$ & $95 \%(81 / 85)$ \\
Side branches & 182 & 20 & 154 & 5 & 3 & $87 \%(20 / 23)$ & $97 \%(154 / 159)$ & $80 \%(49 / 61)$ & $98 \%(154 / 157)$ \\
RCA & 392 & 71 & 299 & 19 & 3 & $96 \%(71 / 74)$ & $94 \%(299 / 318)$ & $79 \%(71 / 90)$ & $99 \%(299 / 302)$ \\
Proximal & 120 & 30 & 81 & 9 & 0 & $100 \%(30 / 30)$ & $90 \%(81 / 90)$ & $77 \%(30 / 39)$ & $100 \%(30 / 30)$ \\
Middle & 103 & 31 & 63 & 8 & 1 & $97 \%(31 / 32)$ & $89 \%(63 / 71)$ & $79 \%(31 / 39)$ & $98 \%(63 / 64)$ \\
Distal & 91 & 8 & 80 & 2 & 1 & $89 \%(8 / 9)$ & $98 \%(80 / 82)$ & $80 \%(8 / 10)$ & $99 \%(80 / 81)$ \\
PDA & 78 & 2 & 75 & 0 & 1 & $67 \%(2 / 3)$ & $100 \%(75 / 75)$ & $100 \%(2 / 2)$ & $99 \%(75 / 76)$ \\
\hline
\end{tabular}

$\mathrm{CX}=$ circumflex coronary artery $\mathrm{FN}=$ false negative; $\mathrm{FP}=$ false positive; $\mathrm{LAD}=$ left anterior descending coronary artery; $\mathrm{LM}=$ left main coronary artery; $\mathrm{PDA}=$ posterior descending artery; $\mathrm{RCA}=$ right coronary artery; $\mathrm{PV}=$ predictive value; $\mathrm{TN}=$ true negative; $\mathrm{TP}=$ true positive.

interval [CI]: 88 to 95$)$, the specificity was $95 \%$ (1,092 of 1,150, 95\% CI: 93 to 96), the positive predictive value was $79 \%$ (216 of $274,95 \%$ CI: 73 to 88 ), and the negative predictive value was $98 \%$ (1,092 of 1,110, 95\% CI: 97 to 99) (Table 1) for the detection of significantly obstructed lesions.

Nineteen percent (268 of 1,384) of all segments were classified as heavily calcified, $31 \%$ (430 of 1,384$)$ as moderately calcified, and 50\% (686 of 1,384) as noncalcified. The diagnostic performance of MSCT coronary angiography for detection of significant obstructive lesions in noncalcified, moderately calcified, and heavily calcified segments is tabulated in Table 2 .

Lesion-by-lesion analysis: false-negative results on MSCT. Eighteen significantly obstructed segments with a mean diameter reduction of $61.1 \pm 7.5 \%$ (range $51 \%$ to $72 \%$ using QCA) were missed on the MSCT scan. The majority of the missed lesions were located in the circumflex coronary artery or in smaller side branches (13 of $18,72 \%)$. Two lesions were missed because of severe calcifications and two because of motion artifacts; the severity of the stenosis was underestimated in the remaining 14 lesions.

Lesion-by-lesion analysis: false-positive results on MSCT. Fifty-eight segments were incorrectly classified as significantly obstructed because of overestimation of the severity of the lesion. Fifteen noncalcified and 17 moderately calcified lesions (mean diameter reduction $41.2 \pm$
$5.1 \%$, range $30 \%$ to $48 \%$ ) were overestimated. Twenty-six heavily calcified lesions were overestimated; in the majority of these lesions conventional angiography revealed only minor wall irregularities. Overestimation of heavily calcified lesions was likely related to beam-hardening artifacts and partial volume effects resulting in blooming of the coronary calcifications.

Vessel-based analysis. The sensitivity for classification of vessels with or without CAD was $94 \%$ (177 of $188,95 \%$ CI: 89 to 95 ); specificity was $91 \%$ (298 of $329,95 \%$ CI: 86 to 93), positive predictive value was $85 \%$ (177 of $208,95 \%$ CI: 79 to 91 ), and negative predictive value was $96 \%$ (298 of 309, 95\% CI: 93 to 98). All significantly obstructed left main coronary arteries $(n=6)$ and total occlusions $(n=60)$ were correctly identified on the MSCT scan.

Patient-based analysis. Multislice spiral computed tomography coronary angiography correctly identified 18 of 21 (86\%) patients without significant stenoses on angiography. No patient with single-vessel disease $(n=44)$ on angiography was incorrectly classified as having no significant coronary disease on MSCT; however, 15 patients were classified as having multivessel disease. Fifty-five patients with multivessel disease $(n=62)$ on angiography were correctly classified on MSCT; the remaining seven patients were classified as having single-vessel disease.

Overall, all patients with a significant stenosis in one or more vessels were correctly classified as patients with CAD.

Table 2. Diagnostic Performance of Multislice Spiral Computed Tomography Coronary Angiography for the Detection of Significant Obstructive Lesions

\begin{tabular}{lccccccccc}
\hline \multicolumn{1}{c}{ Segments } & N & TP & TN & FP & FN & Sensitivity & Specificity & Positive PV & Negative PV \\
\hline Non-calcified & 686 & 60 & 602 & 15 & 9 & $87 \%(76-97)$ & $98 \%(96-98)$ & $80 \%(69-91)$ & $90 \%(97-99)$ \\
Moderately calcified & 430 & 64 & 342 & 17 & 7 & $90 \%(80-97)$ & $95 \%(92-97)$ & $79 \%(68-90)$ & $98 \%(95-99)$ \\
Heavily calcified & 268 & 92 & 148 & 26 & 2 & $98 \%(92-94)$ & $85 \%(78-90)$ & $78 \%(69-86)$ & $99 \%(95-99)$ \\
Overall & 1,384 & 216 & 1,092 & 58 & 18 & $92 \%(88-95)$ & $95 \%(93-96)$ & $79 \%(73-88)$ & $98 \%(97-99)$ \\
\hline
\end{tabular}

Noncalcified = complete absence of coronary calcification; moderately calcified = Small isolated eccentric calcified deposits; Heavily calcified = Large deposits of calcium expanding longitudinally along the coronary wall, causing partial volume effects and beam-hardening artifacts. Abbreviations as in Table 1 . 


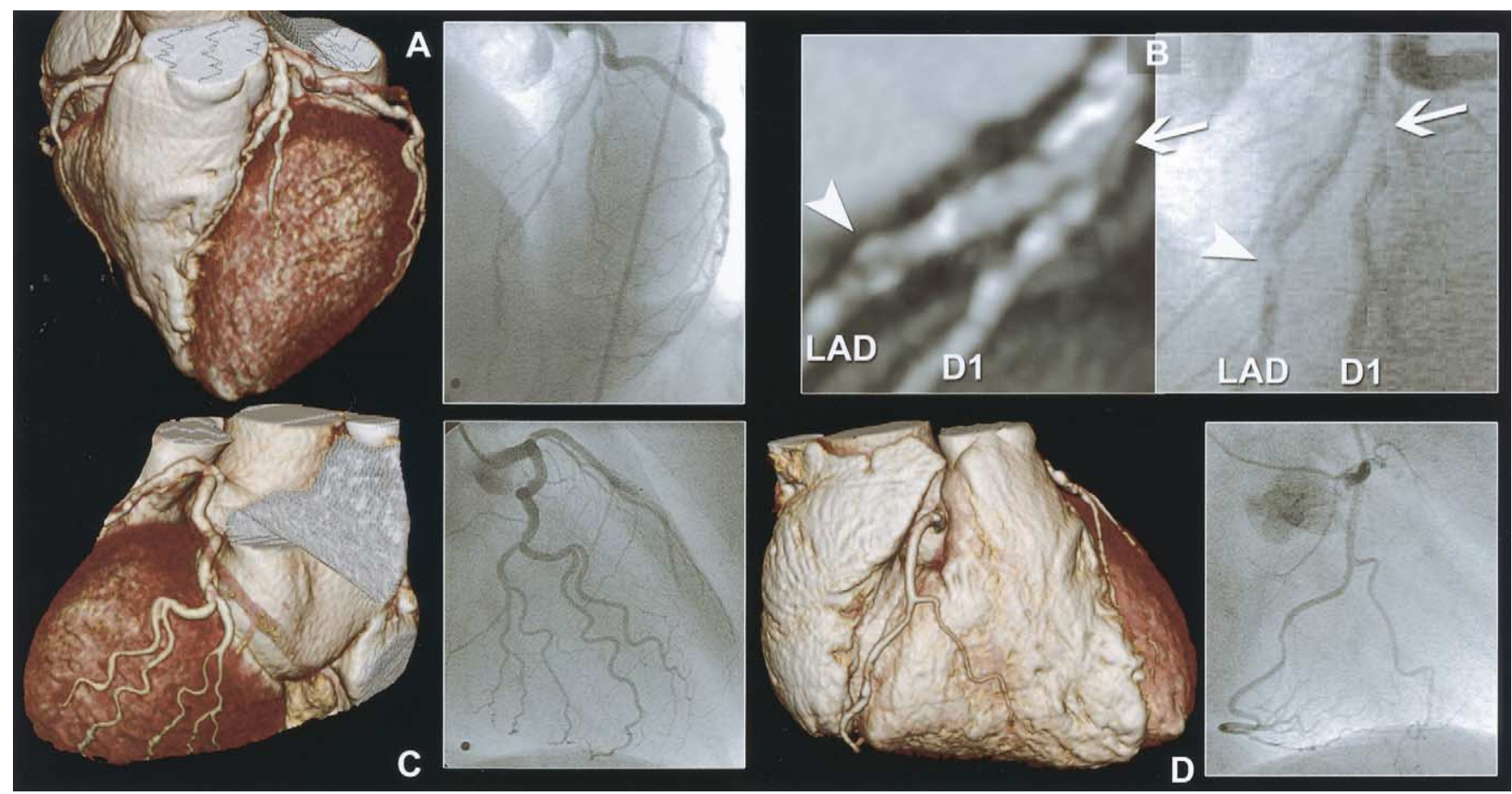

Figure 1. Volume-rendered multislice spiral computed tomography images (left) and corresponding conventional angiography images (right) of the left anterior descending (LAD) (A), circumflex (C), and right coronary artery (D) in a 69-year-old woman with stable angina and a positive bicycle test. Significant lesions were found in the proximal part of the LAD coronary artery (arrowhead) and first diagonal (D1) (arrow), which are demonstrated on the inlay (B).

Sensitivity for classification of patients with or without CAD was $100 \%$ (106 of 106, 95\% CI: 96 to 100), specificity was $86 \%$ (18 of $21,95 \%$ CI: 63 to 96), positive predictive value was $97 \%$ (106 of 109, 95\% CI: 92 to 98), and negative predictive value was $100 \%$ (18 of $18,95 \%$ CI: 81 to 100 ).

\section{DISCUSSION}

Complete visualization of all clinically important coronary segments is a prerequisite for MSCT coronary angiography to become an accepted clinical tool for the assessment of patients with suspected CAD. Previous studies in relatively small cohorts showed high sensitivity and specificity for the detection of significant obstructive lesions $(2,3)$. The major finding of the present study, which compared MSCT with $\mathrm{QCA}$, is that 16-row MSCT is a robust tool for assessing the presence of significantly obstructed coronary artery in the clinically important part of the coronary tree (Figs. 1 and 2). All MSCT segments corresponding to angiographic segments with a diameter above $2 \mathrm{~mm}$ on QCA were analyzed, without any prespecified exclusion criteria based on the quality of the MSCT images. This resulted in 10.9 \pm 1.9 available segments per patient. For the segmentbased analysis, both the overall sensitivity and specificity were found to be above $90 \%$ ( $92 \%$ and $94 \%$, respectively). Image quality was classified as poor in $7 \%$ of the segments. However, these segments were included in the comparative analysis with QCA.

The current-generation 16-slice scanners have a higher spatial and temporal resolution when compared with previ- ous scanners, resulting in an improvement in the diagnostic accuracy of MSCT angiography (7-10).

One-half of the segments contained calcified deposits in the coronary wall. Calcifications are high-density structures causing beam-hardening artifacts and partial volume effects on computed tomography. These artifacts have an important impact on the evaluation of calcified lesions. Only two lesions in the present study were missed because of severe calcifications, but 26 heavily calcified lesions were overestimated. The majority of these lesions appeared as wall irregularities on conventional angiography.

Another 32 overestimated lesions with a mean diameter reduction of $40 \%$ on QCA (range between $30 \%$ and $48 \%$ ) were noncalcified or moderately calcified. This probably reflects the semiquantitative nature of the evaluation of coronary lesions with MSCT.

One-third of the missed $\geq 50 \%$ lesions were located in the circumflex and a further one-third in smaller side branches. The circumflex coronary artery is more difficult to evaluate, probably because of its tortuous course and overlapping structures, whereas the relatively high number of missed lesions in smaller side branches suggest a lower diagnostic performance of MSCT angiography in branches toward the threshold of $2 \mathrm{~mm}$.

All patients with at least one significant obstruction on QCA were correctly classified as having CAD with MSCT. This suggests that the current generation of scanners may be a suitable tool to triage patients with stable angina who are 


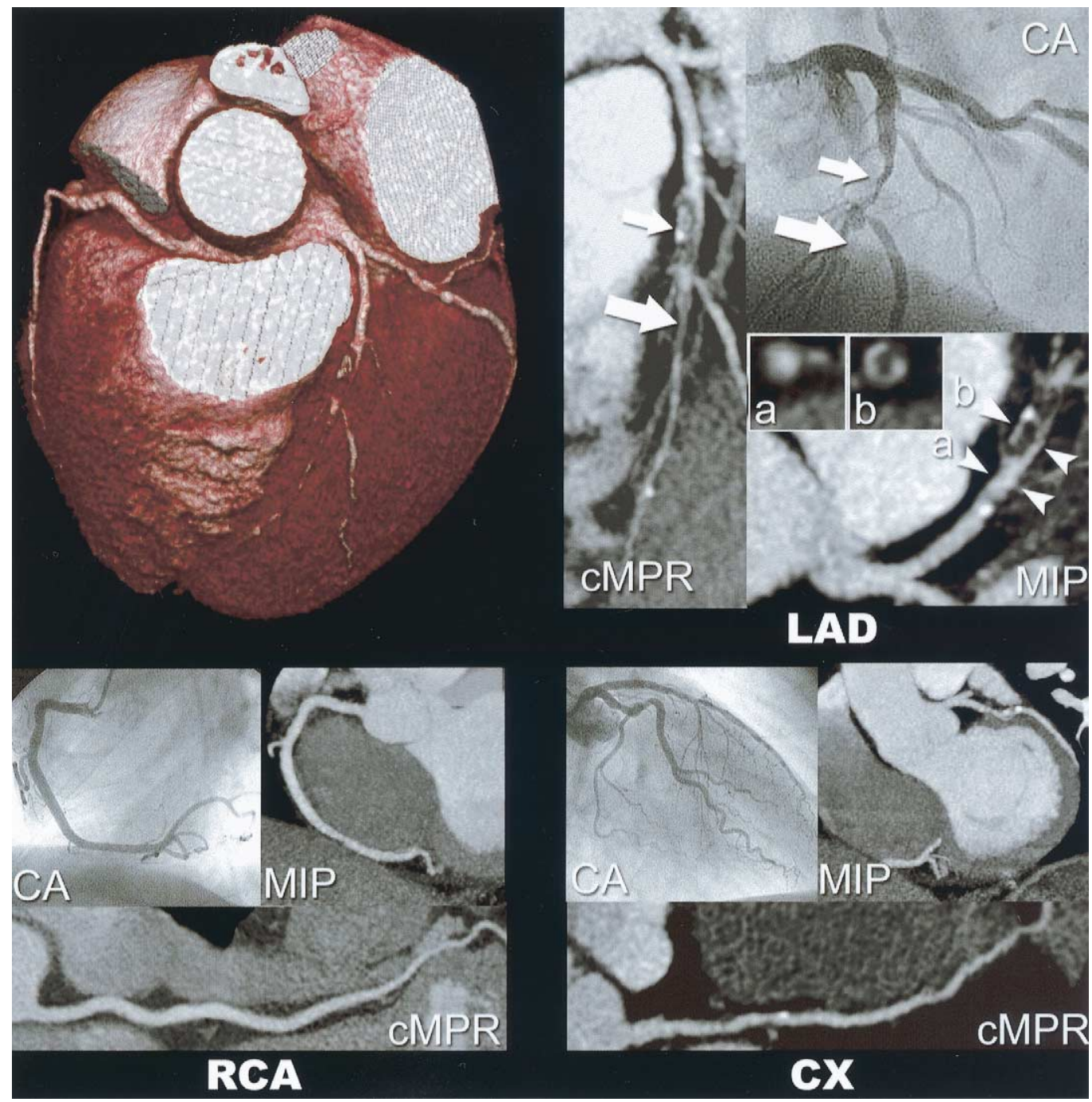

Figure 2. Colored image $=$ volume rendered multislice spiral computed tomography (MSCT) image providing an overview of the anatomy of the main coronary arteries. Black and white images $=$ MSCT (curved multiplanar reconstructions [cMPR] and maximum intensity projections $[\mathrm{MIP}]$ ) and conventional coronary angiography (CA) images of the four main coronary arteries (left main/left anterior descending [LAD], circumflex coronary artery $[\mathrm{CX}]$, right coronary artery [RCA]) of a single patient. The small arrows highlight a high-grade stenosis and the large arrows an occlusion located at the midpart of the LAD. The arrowheads indicate cross-sectional images proximal (inlay a) and within the occlusion (inlay b). Inlay b shows calcified (displayed as white) and noncalcified (displayed as black) plaque tissue components.

being considered for revascularization. Further studies are needed to determine the role of this technique in the clinical workup of patients with other presentations of coronary disease or who are being evaluated to exclude significant disease.

Study limitations. Only patients with stable angina were included in this study. Whether a broader group of patients might benefit from this technique (excluding patients with severe arrhythmia) must be explored, but there are no reasons to believe that results would differ in patients with acute coronary syndromes.

In our study we noted that the sensitivity of detection for noncalcified obstructive lesions tended to be lower. This is caused by the fact that these lesions have a relatively low tissue contrast and may be missed, especially in small vessel segments. This may be problematic in younger patients with a lower likelihood of lesion calcification. Dedicated auto- matic software that is able to detect and calculate the degree of coronary stenoses would improve diagnostic accuracy.

The high radiation exposure during MSCT coronary angiography, which is reported between 6.7 and $13.0 \mathrm{mSv}$ $(5,11,12)$, remains a matter of concern. Further fine-tuning of the prospective $\mathrm{X}$-ray tube current modulation and development of new features lowering radiation exposure is highly desirable.

Conclusions. Multislice spiral computed tomography coronary angiography permits reliable detection of CAD in a population of patients in sinus rhythm with stable angina.

Reprint requests and correspondence: Dr. Pim J. de Feyter, Erasmus Medical Center, Department of Cardiology and Radiology, Thoraxcenter, Bd 410, P.O. Box 2040, 3000 CA Rotterdam, Netherlands. E-mail: p.j.defeyter@erasmusmc.nl. 


\section{REFERENCES}

1. Flohr TG, Schoepf UJ, Kuettner A, et al. Advances in cardiac imaging with 16-section CT systems. Acad Radiol 2003;10:386-401.

2. Nieman K, Cademartiri F, Lemos PA, Raaijmakers R, Pattynama $\mathrm{PM}$, de Feyter PJ. Reliable noninvasive coronary angiography with fast submillimeter multislice spiral computed tomography. Circulation 2002;106:2051-4.

3. Ropers D, Baum U, Pohle K, et al. Detection of coronary artery stenoses with thin-slice multi-detector row spiral computed tomography and multiplanar reconstruction. Circulation 2003;107:664-6.

4. Jakobs TF, Becker CR, Ohnesorge B, et al. Multislice helical CT of the heart with retrospective ECG gating: reduction of radiation exposure by ECG-controlled tube current modulation. Eur Radiol 2002;12:1081-6.

5. Trabold T, Buchgeister M, Kuttner A, et al. Estimation of radiation exposure in 16-detector row computed tomography of the heart with retrospective ECG-gating. Rofo Fortschr Geb Rontgenstr Neuen Bildgeb Verfahr 2003;175:1051-5.

6. Austen WG, Edwards JE, Frye RL, et al. A reporting system on patients evaluated for coronary artery disease. Report of the Ad Hoc
Committee for Grading of Coronary Artery Disease, Council on Cardiovascular Surgery, American Heart Association. Circulation 1975;51:5-40.

7. Nieman K, Oudkerk M, Rensing BJ, et al. Coronary angiography with multi-slice computed tomography. Lancet 2001;357:599-603.

8. Achenbach S, Giesler T, Ropers D, et al. Detection of coronary artery stenoses by contrast-enhanced, retrospectively electrocardiographicallygated, multislice spiral computed tomography. Circulation 2001;103: 2535-8.

9. Knez A, Becker CR, Leber A, et al. Usefulness of multislice spiral computed tomography angiography for determination of coronary artery stenoses. Am J Cardiol 2001;88:1191-4.

10. Vogl TJ, Abolmaali ND, Diebold T, et al. Techniques for the detection of coronary atherosclerosis: multi-detector row CT coronary angiography. Radiology 2002;223:212-20.

11. Morin RL, Gerber TC, McCollough CH. Radiation dose in computed tomography of the heart. Circulation 2003;107:917-22.

12. Hunold P, Vogt FM, Schmermund A, et al. Radiation exposure during cardiac CT: effective doses at multi-detector row CT and electron-beam CT. Radiology 2003;226:145-52. 